\title{
An IoT Based Health Care and Patient Monitoring System to Predict Medical Treatment using Data Mining Techniques: Survey
}

\author{
Aieshwarya. B. Chavan Patil ${ }^{1}$ \\ PG Student, Computer Engineering, Pune Institute of Computer Technology, Pune, India ${ }^{1}$
}

\begin{abstract}
The majority of patients in the hospital are non-ambulatory and would benefit from predictive and personalized monitoring system. Technology plays the major role in healthcare not only for sensory devices but also in communication, recording and display device. It is very important to monitor various medical parameters and post operational days. Hence the latest trend in Healthcare communication method using IOT is adapted. Internet of things serves as a catalyst for the healthcare and plays prominent role in wide range of healthcare applications. In this project the AVR-328 microcontroller(Arduino board) is used as a gateway to communicate to the various sensors such as temperature sensor, heartbeat sensor, ECG sensor, sensor for keeping a track of drip levels(blood or saline). The microcontroller picks up the sensor data and sends it to the network through Wi-Fi and hence provides real time monitoring of the health care parameters for doctors. The data can be accessed anytime by the doctor. The controller is also connected with buzzer to alert the caretaker about variation in sensor output. At the time of extremity situation alert message is sent to the doctor through the android application connected to the cloud server. Hence quick provisional medication can be easily done by this system. Also an additional subsystem is provided for prediction of heart disease for the patient based on his/her health parameters. This system is efficient with low power consumption capability, easy setup, high performance and time to time response.
\end{abstract}

Keywords: IOT (Internet Of Things), Data mining, Arduino kit, Health Parameters, Cloud storage, Motion detection, Heart Disease Prediction.

\section{INTRODUCTION}

Health is one of the global challenges for humanity. Studies have shown that 30 percent of patients with a According to the constitutions of World Health discharge diagnosis of heart failure are readmitted at least Organization (WHO) the highest attainable standard of once within 90 days with readmission rates ranging from health is a fundamental right for an individual[1].

In hospitals there are provisions for continuous monitoring of patients. Their ECGs, heartbeat are continuously monitored. There is no provision to check the parameters when they return to home. And hence there is a chance that the disease may return again. Patient's data (temperature, heart rate, ECG, position) will be frequently measured and sent to server. Period of sending (say every $1 \mathrm{~min})$ can be set. Monitoring person learns patient specific threshold. Say the regular body temperature of a patient is $24^{\circ} \mathrm{C}$ whereas one person feels feverish if his body temperature is $32^{\circ} \mathrm{C}$. By employing an averaging technique over a relatively long time, Observer can learn these thresholds for patients [1]. Using Android Application in doctors smart phone, doctor can view his patients health status. When any of the parameter goes beyond the threshold value he will get an alert notification. Using Andriod Application in patients or his caretakers smart phone the patient can view his health status. Early detection and diagnosis of potentially fatal physiological conditions such as heart attack require continuous monitoring of patients health following transfer from hospital to home. $25 \%$ to $54 \%$ within 3 to 6 months. In response to these types of needs, health monitoring systems are being proposed as a low cost solution. Such a system consists of physiological data that stores, process and communicate through a local manner such as smart phones, personal computers. Such systems should satisfy strict safety, security, reliability, and long-term real time operation requirements [2].

In the proposed system we present a health monitoring system that uses the sensors for collecting data from patients, intelligently predicts patient's health status and provides feed- back to doctors through their mobile devices having android application. The patients will participate in the health care process by their mobile devices and thus can access their health information from anywhere any time. Today Internet has become one of the important part of our daily life. It has changed how people live, work, play and learn. Internet serves for many purpose educations, finance, Business, Industries, Entertainment, Social Networking, Shopping, ECommerce etc .The next new mega trend of Internet is Internet of Things (IOT). Visualizing a world where several objects can sense, communicate and share 
information over a Private InternetProtocol (IP) or Public Networks. The interconnected objects collect the data at regular intervals, analyse and used to initiate required action, providing an intelligent network for analyzing, planning and decision making. This is the world of the Internet of Things (IOT).

The IOT is generally considered as connecting objects to the Internet and using that connection for control of those objects or remote monitoring. But this definition was referred only to part of IOT evolution considering the machine to machine market today. But actual definition of IOT is creating a brilliant, invisible network which can be sensed, controlled and programmed. The products developed based on IOT include embedded technology which allows them to exchange information, with each other or the Internet and it is assessed that about 8 to 50 billion devices will be connected by 2020. Since these devices come online, they provide better life style, create safer and more engaged communities and revolutionized healthcare. The entire concept of IOT stands on sensors, gateway and wireless network which enable users to communicate and access the application/information.

\section{EXISTING TECHNIQUES AND SYSTEMS FOR HEALTH CARE AND PREDICTION OF HEART DISEASE}

\section{A) U-HEALTHCARE SYSTEM:}

Bourouis [6] has discussed about ubiquitous healthcare system. It is an emerging technology that provides easy healthcare service to patients, and convenient to diagnose patients health condition. It provides increase in accuracy, efficiency and availability of medical treatment so people can monitor their health by an online medium without visiting the hospital or clinic.

B) Health CareKey Management Proposed Solution:

Yuce[3] has discussed about Healthcare monitoring system where the patient carries a set of WBAN devices and the patient is ambulatory, but is always on the reach of the gateway that binds the sensors with the medical central unit. These devices communicate remotely through the internet with a monitoring medical central unit installed in a hospital or clinic responsible for monitoring patients health status. [7,8].Caregivers can connect through the internet via computers or mobile devices to the medical central unit to supervise patient's data processed and analyzed by dedicated applications. The object is to address the data privacy of the mobile WBAN sensors exchanged with the remote medical central unit.

C) Security For Healthcare Systems:

Kumar [4] has discussed about security for healthcare techniques. Introducing new technologies in healthcare system without considering security makes patient privacy vulnerable. The physiological data or report of an each and every individual patient is highly sensitive. The wireless medical sensors produce or collected large data which must be secured from security attacks. The success of healthcare applications mainly depends on patient security and privacy.

D) Real Time Wireless Health Monitoring:

Amna Abdullah[8] has discussed the implementation of the Wireless Health Monitoring System and its components are ECG Electrodes, LM35 Temperature Sensor, Blood Pressure Sensor, Blood Glucose Sensor, Microsoft Pro Tablet. The system operates as the medical professional used three electrodes of ECG on the patients body and connect the arduino shield with a temperature sensor, a blood glucose level sensor and a blood pressure sensor.From the arduino shield they connect a wireless node and the Tablet or the Smartphone that has Lab View software running on it to take reading of the patients physiological data.The data are saved according to the time and presented in a report format and the data is then published in the internet by using tablet or smartphone so that the patients report can be accessed by the authorized healthcare persons from remote locations at any time.

\section{E) Classification Algorithms For Prediction Of Heart} Disease :

P.Deepika[9] has provided different types of classification techniques in the field of Heart Disease prediction that include mainly five different classification algorithms namely Classification by decision tree, Bayesian Classification, Neural Networks, Support vector machine, Classification based on association are out of which Neural networks give $100 \%$ accuracy while decision trees and naive bayes provide $99.62 \%$ and $90.74 \%$ accuaracy according to K.Thenmozhi[10].

\section{III.PROPOSED APPROACH}

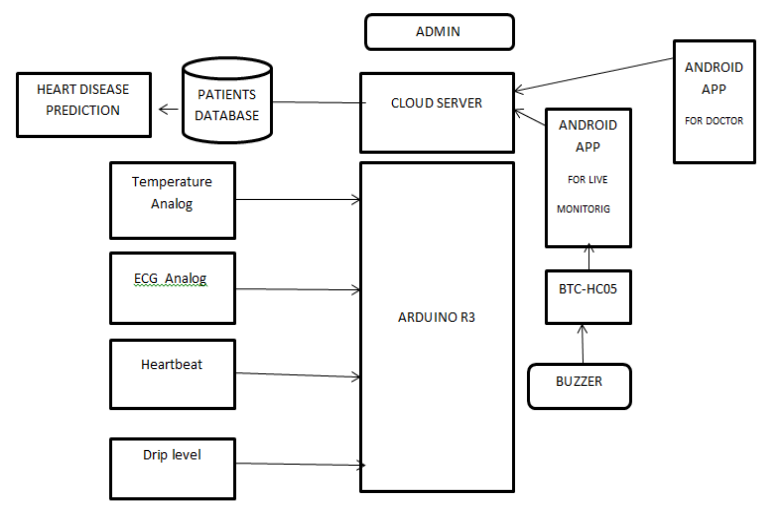

Fig. 1. Architecture of proposed system

\section{IV.CONCLUSION}

By using the system the healthcare professionals can monitor, diagnose, and advice their patients all the time. The health parameters data are stored and published online. Hence, the healthcare professional can monitor their patients from a remote location at any time. Our system is simple and patient's data can be easily accessed 
by usage of NFC tags.It is time saving as well as it considers the security parameter for the system. The Future work of the project is very essential in order to make the design system more advanced. In the designed system the enhancement would be connecting more sensors to internet which measures various other health pameters and would be beneficial for patient monitoring i.e. connecting all the objects to internet for quick and easy access. Establishing a Wi-Fi mesh type network to increase in the communication range.

\section{ACKNOWLEDGMENT}

I sincerely convey my gratitude to my guide Prof. S. S. Sonawane, Department of Computer Engineering for her constant support, providing all the help, motivation and encouragement from beginning till now to make this work a perfect one. I also sincerely thank our Dissertation Coordinator Dr. Archana Ghotkar and Head of Computer Engineering Department, Dr. R. B. Ingle for their support. I am also grateful to my friends for all their help and support. Above all I would like to thank my parents for their wonderful support and blessings, without which I would not been able to accomplish my achievement.

\section{REFERENCES}

[1] Global Challenges for Humanity available at http://www.millenniumproject.org/millennium/challenges.html

[2] Lei Clifton, David A. Clifton, Marco A. F. Pimentel, Peter J. Watkinson, and Lionel Tarassenko (2014), Predictive Monitoring of Mobile Patients by Combining Clinical Observations with Data From Wearable Sensors, IEEE Journal of Biomedical and Health Informatics, Vol. 18, No. 3,May, pp. 722-730.

[3] Yuce, M. R.(2010) Implementation of wireless body area networks for health care systems, Sensor and Actuators A:Physical, Vol. 162, No. 1,July, pp. 116-129.

[4] Kumar P., Lee, S., Lee, H.: E-SAP: efficient-Strong authentication protocol for healthcare applications using wireless medical sensor networks. Sensors1, 1625-1647 (2012).

[5] Intelligent Heart Disease Prediction System Using Data Mining Techniques Sellappan Palaniappan, Rafiah Awang,978-1-42441968-5/08/\$25.00 @2008 IEEE.

[6] Bourouis, A., Feham, M., and Bouchachia, A.(2011), Ubiquitous Mobile Health Monitoring System for Elderly (UMHMSE), International Journal of Computer Science and Information Technology, Vol.2, No.

[7] Bhoomika.B.K, Dr. K N Muralidhara, Smart Healthcare Monitoring System Based on Iot, nternational Journal on Recent and Innovation Trends in Computing CommunicationVolume: 3 Issue,JRITCC | July 2015.

[8] Amna Abdullah, Asma Ismael, Aisha Rashid, Ali Abou-ElNour, and Mohammed Tarique, Real Time Wireless Health Monitoring Application using Mobile Devices, International Journal of Computer Networks \& Communications (IJCNC) Vol.7, No.3, May 2015.

[9] P.Deepika,P.Vinothini,Research Paper Heart Disease Analysis And Prediction Using Various Classification Models, Volume : 4 | Issue : 3 | Mar 2015 , ISSN - 2250-1991.

[10] K.Thenmozhi,Different Data Mining Techniques Involved in Heart Disease Prediction,IJSRInternational Journal Scientific Research volume : 3 | Issue : 9 | September 2014. 